\title{
And now we are one
}

\author{
As a newcomer to the community, Nature Astronomy covered regional and international scientific and \\ societal issues, discoveries and advances in its first year. We anticipate an equally if not more exciting year to come.
}

W elcome to a new volume of Nature Astronomy. First of all, we thank all of you, the contributors who helped us launch the journal twelve months ago and who helped fill our pages with exciting discoveries, insightful commentaries and thoughtful scientific discourse throughout 2017. Authors are always visible, of course, but the reviewers are less so. We are grateful to them all, for without them there would be no journal.

Within the astronomy-related publishing sphere, there are numerous excellent and established journals. Authors have a wide choice of where to submit their work based on a multitude of factors: speed, reliability, visibility, quality, value for money and so forth. Our aim has always been to select the most significant advances and make them accessible to as wide an audience as possible. To this end, our team of full-time editors encourages authors to put their work in context and explain technical terms (not avoid them; we do require all the details). Besides text editing by editors and copy editors, our dedicated art editors improve the clarity and presentation of figures and tables.

In addition to the original research Letters and Articles that we publish, we provide a forum for the discussion of topical issues in our magazine-style 'front half'. By travelling to visit institutions and attending conferences, we get to hear about all sorts of exciting, or sometimes concerning, developments that deserve to be broadly but concisely communicated to our scientific community. Often, we commission Comments, Perspectives and Reviews based on particularly interesting talks or conversations. Other times, we rely on our editorial discretion to highlight issues that we feel may warrant more discussion and attention from the community. When there is a critical mass, we can publish a Focus on a specific topic. Take, for instance, our Focus on gender equity in astronomy. The germination of the idea came with the submission of 'Quantitative evaluation of gender bias in astronomical publications from citation counts' by Neven Caplar, Sandro Tacchella and Simon Birrer. Using machine-learning techniques to account for non-gender-specific attributes, these authors concluded that papers with female first authors receive $10 \%$ fewer citations. With this paper as an anchor, we invited several authors to comment on the different manifestations of this persistent discrimination. Together they paint a worrying picture, but one that the public is finally recognizing and hopefully will begin to meaningfully address immediately.

Another Focus celebrated the 50th anniversary of the discovery of pulsars. This Focus could easily have been a retrospective, given the activity that followed the first observation of a pulsar; instead, a few historical pieces are complemented by the latest research into the physics of pulsars and the applications of pulsar arrays. We also love receiving suggestions for content as well. In fact, our dark matter Insight started with one suggested Review and blossomed into nine pieces that we published jointly with Nature Physics. Be it a longer format Review or a concise opinion in the form of a Comment or Correspondence to the Editor, we are happy to receive your contributions.

At Nature Astronomy, we begin 2018 by focusing on the future. Specifically, we look at different career paths for astronomers, astrophysicists and planetary scientists. If, following the latest job-hunting season, your resolution is to start a career outside of academia, we hope that we can help. But why leave? Many professional astronomers, astrophysicists and planetary scientists do what they do out of interest and passion. Being a researcher or professor or telescope operator is a vocation in its full sense, much more so than an occupation. But this emotional driver - and the fact that the supply of $\mathrm{PhD}$-qualified labour grows year on year - means that those working in academia on short-term, temporary contacts (the majority) are underpaid and lacking in benefits compared to those in industry. In addition, they usually undergo periodic relocation, often internationally, and furthermore must deal with the emotional and mental pressures of working with little job security in an increasingly competitive environment. Those who do not get tenure sometimes consider themselves failures or, worse, are branded failures by others. However, in this issue of Nature Astronomy we hear from many former astronomers and planetary scientists who can attest that there is life after academia, and more than that, that life can be better after academia.

In this Focus on careers, we hear the in-depth experiences of five former academic astronomers or planetary scientists who are now following different career paths: a planetary scientist who formed her own company with colleagues in order to continue their research program outside of the 'ivory tower'; an astrochemist who became a journal editor; an astronomer who became a data scientist; an astronomer who turned to science writing and journalism; a further astronomer who moved into education and public outreach. We also publish the results of a survey on the nonacademic career paths of astronomers, adding hard numbers to the personal experiences. Finally, we asked eleven former astronomers now working in diverse fields the same five quick-fire questions about their occupational transitions. The overall message from this compilation is both worrying for the current state of academia but also overwhelmingly positive for the alternatives; as Alaina Levine says in her Comment: "Your career prospects are bright ... your career options are immense ... your professional triumph and bliss are attainable ... the cosmos is your kingdom!"

And what more could we possibly add to that? If nothing else, we hope that seeing other people's success will help remove the stigma of not getting a tenured academic position, prompting astronomers to actively choose alternative careers, rather than feeling forced to do so. If you have any ideas on other issues to be covered, or would like to offer feedback on our published content, please do get in touch with us at natureastro@nature.com. We look forward to another year of discoveries and innovations.

Published online: 2 January 2018 https://doi.org/10.1038/s41550-017-0370-3 